\title{
A new role for mathematics in empirical sciences
}

\author{
Atoosa Kasirzadeh (University of Toronto \& Australian National University) \\ Forthcoming in Philosophy of Science
}

\begin{abstract}
Mathematics is often taken to play one of two roles in the empirical sciences: either it represents empirical phenomena, or it explains these phenomena by imposing constraints on them. This paper identifies a third and distinct role which has not been fully appreciated in the literature, and may be pervasive in scientific practice. I call this the "bridging" role of mathematics, according to which mathematics acts as a connecting scheme in our explanatory reasoning about why and how two different descriptions of an empirical phenomenon relate to each other. I discuss two bridging roles appearing in biological and physical explanations.
\end{abstract}




\section{Introduction}

Some philosophers maintain that either mathematics is merely representational of the empirical phenomena in scientific explanations, or it has a non-representational, constraining-explanatory role. The former is uncontroversial. As an integral part of scientific explanations, mathematics plays a significant role in idealized representations of the empirical world. In contemporary literature this role is often analyzed in two ways: either by appealing to the so-called mapping account of Pincock (2004, 2007), which suggests that there is some kind of structural morphism between mathematics and the empirical world, or by the inferential account of Bueno and Colyvan (2011) and Bueno and French (2018), which along with structural morphism emphasizes pragmatic and context-dependent features in applying mathematics to the empirical world. ${ }^{1}$

In contrast, some philosophers have promoted a genuinely explanatory role for mathematics in the empirical sciences. In one of its promising versions, Lange $(2012,2017)$ argues that mathematics can factor into explanations by constraining the empirical world. For instance, in explaining why a mother cannot divide 23 whole strawberries evenly among her three children, the mathematical fact that 23 cannot be divided evenly by 3 constrains her action and explains her inability. There are certainly additional accounts of how mathematics might explain physical phenomena, e.g., Batterman (2009), but I will primarily restrict myself to the representational and Lange's constraining accounts in order to keep this paper to a manageable length. In section 3, however, some alternative accounts will be briefly mentioned.

${ }^{1}$ Earlier versions of Pincock's $(2004,2007)$ view can be found in standard mathematics textbooks such as Stewart $(2008,24)$, and also in the classic work on measurement by Krantz et al. (1971). 
Both of these views about the roles of mathematics, representing and constraining, have much to recommend then, but as I will show, they are not exhaustive. In this paper, I identify a third and distinct role which I will call the "bridging" role for mathematics in explanations. According to this role, mathematics acts as a connecting scheme in our explanatory reasoning about why and how two different descriptions of an empirical phenomenon relate to each other. In section 2, I describe the representational and the constraining-explanatory roles of mathematics. In section 3, I propose that the bridging role of mathematics is distinct from both the representational and the constraining-explanatory roles. In support of my proposal, I present a case study analyzing a scientific explanation of color pattern formation by mathematical biologists. Subsequently, I show why Bueno and Colyvan's (2011) and Bueno and French's (2018) framework for the applicability of mathematics cannot fully accommodate the bridging role of mathematics in this explanation. Hence, I revise their framework to fulfill this task. In section 4, I argue that the bridging role is general enough, and it is found in other cases of explanation, one of which is a familiar historical example. In particular, I will discuss how this role appeared in an explanation of why and how two variant descriptions of quantum phenomena were found to have empirically-significant, mathematical equivalence. Section 5 concludes the paper.

\section{The Representational and Constraining-explanatory Roles}

Advocates of the representational role of mathematics in explanations, such as Pincock (2004, 2007), Bueno and Colyvan (2011), and Bueno and French (2018) believe that mathematics plays a role in empirical sciences in virtue of some structural morphism between an abstract, formal structure and its appropriate empirical counterpart. The role of Euler's theorem in 
explaining why no one can cross all the bridges of Königsberg only once before returning to their starting point is a classic example. The explanation bears on the specific configuration of the bridges and paths that exhibit the structure of a non-Eulerian graph. The idea is that given the topological structure of the actual bridges and our abstract mathematical knowledge about the properties of Eulerian and non-Eulerian paths, we find a mapping relation between the mathematical structure and the empirical phenomenon. It is exactly in virtue of this structural mapping that mathematics becomes explanatory.

Pincock $(2004,2007)$ develops his mapping account according to the widespread view that the applicability of mathematics to the empirical world is due to sharing some structural similarity between mathematics and the empirical phenomenon of interest. The existence of such structural similarity sufficiently accommodates the applicability of a given mathematical structure to the empirical phenomenon.

Bueno and Colyvan (2011) and Bueno and French (2018, Chapter 2) introduce the inferential account for the applicability of mathematics by expanding upon Pincock's structural-mapping account. Along with structural morphism, they incorporate some pragmatic elements that are relevant to mathematical explanation and idealization, and are necessary for the applicability of mathematics to the empirical world. The main claim of Bueno and Colyvan (2011) and Bueno and French (2018) is that when certain features of the empirical world are embedded into a mathematical structure, we can obtain inferences which might otherwise be impossible (or at least, extremely difficult) to draw. This account proceeds in three steps: (i) immersion: establishing a mapping between a mathematical structure $M_{1}$ and a characterization of an empirical phenomenon $L_{1}$; (ii) derivation: drawing mathematical consequences $M_{2}$ from $M_{1}$; (iii) interpretation: interpreting $M_{2}$ back to a descriptive level of the empirical phenomenon $L_{2}$ by establishing some sort of mapping relation. Step (ii) is empty 
of pragmatic considerations; as Bueno and Colyvan $(2011,353)$ and Bueno and French $(2018$, 52-3) put it: "The second step consists in drawing consequences from the mathematical formalism, using the mathematical structure obtained in the immersion step." Hence, according to the inferential framework, $M_{2}$ is always a purely mathematical consequence of $M_{1}$. On the other hand, the steps (i) and (iii) encode some pragmatic and context-sensitive features, such as what to map and interpret, in applying mathematics to the empirical world. ${ }^{2}$ This account is illustrated in figure 1.

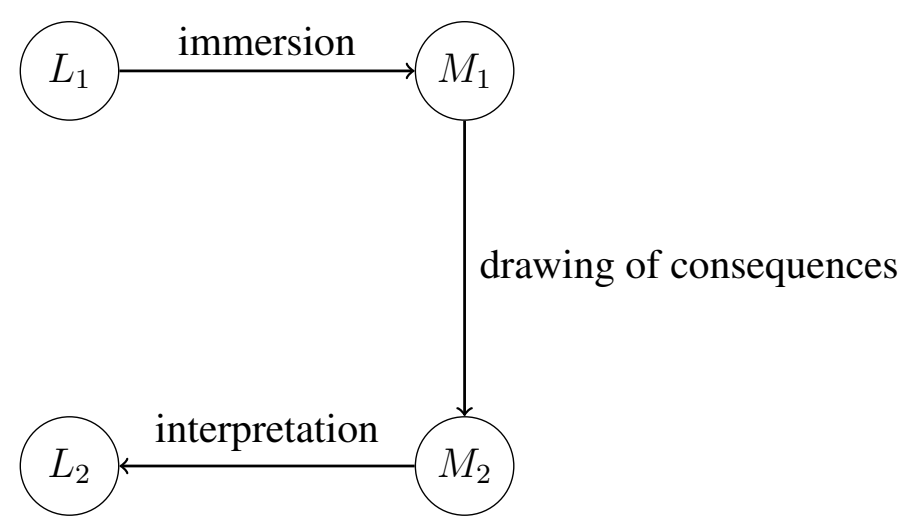

Figure 1: The inferential account.

In this paper, I am concerned with cases in which a given empirical phenomenon of interest is characterized at two distinct descriptive levels $L_{1}$ and $L_{2}$. Understanding how and why the two levels connect then would be a legitimate explanatory question. For each of the $L_{1}$ and $L_{2}$, the mathematical representations $M_{1}$ and $M_{2}$ are distinctly obtained. Each representation illustrates a mapping between a mathematical structure and the empirical phenomenon. To explain why the two descriptive levels $L_{1}$ and $L_{2}$ connect, the characteristics of the relation ${ }^{2}$ In the rest of the paper, I only focus on Bueno and Colyvan's (2011) and Bueno and French's (2018) representational account, as their account extends Pincock's mapping account by incorporating pragmatic considerations. 
between the two mathematical representations $M_{1}$ and $M_{2}$ should be examined. The exploration of why and how the two descriptive levels connect is especially interesting, because the mathematical representations are limited to a particular scientific discourse, mathematical/computational biology (section 3), and physics (section 4). In other words, the investigation of the relationship between the two representations pertains to a specific group of scientists. The second step of the inferential account, as illustrated in figure 1, provides resources to capture the derivation of mathematical consequences $M_{2}$ from the mathematical representation $M_{1}$. Although in some cases the link between the representations $M_{1}$ and $M_{2}$ is explained in terms of consequence derivation, I will shortly discuss the shortcomings of step (ii). I then propose how these shortcomings can be overcome by recognizing the bridging role of mathematics.

Before I discuss my point further, I would briefly clarify why the bridging role of mathematics is also distinct from the constraining-explanatory role. Defenders of the genuinely constraining role such as Lange $(2009,2012,2017)$ attribute a constraining strength to mathematics. On this view, Euler's theorem becomes explanatorily relevant, because it imposes mathematical constraints on how things can be in the empirical world. Lange's account, of course, requires commitment to a particular relation among different constraining strengths; mathematics being more constraining than the empirical laws of nature. Hence, Lange's view about the explanatory role of mathematics may be appealing to those who share his theory of constraining strengths, but controversial to those who reject that theory. The bridging role of mathematics, as I will discuss shortly, is compatible with this constraining-explanatory role, but it does not need to be. The two examples presented in sections 3 and 4 primarily examine some problems for the mapping and the inferential approaches. The reason that I have very briefly mentioned Lange's proposal, as a prominent exemplar for the explanatory role of mathematics, 
is to show that in addition to the bridging role proposed in this paper, there are other philosophical views challenging the idea that mathematics merely plays a representational role in scientific explanations. In other words, I aim to highlight that my proposal is not the only one challenging the representational view.

In the rest of the paper, I provide two case studies to illustrate how the bridging role works in scientific practice. In section 3, I discuss how mathematical biologists appeal to the bridging role to explain the relation between a macro-level and a micro-level characterization of an empirical phenomenon, namely the pattern formation on animal skins. I show how using new parts of mathematics, independent from the mathematics employed for capturing the similarities with the empirical phenomenon of interest, helps obtaining the micro-level representation from the macro-level mathematical representation. In section 4, I illustrate the bridging role of mathematics in explaining the empirical adequacy of two mathematical representations of quantum phenomena. The two case studies reveal how obtaining approximate representations, using bridging mathematical facts, is a very different activity when compared to drawing of consequences as suggested in step (ii) of Bueno and Colyvan's (2011) and Bueno and French's (2018) inferential framework. The examples discussed in sections 3 and 4 show that the relation between the two mathematical representations is not necessarily a consequence derivation. The relation in question can be an approximation relation (section 3) or an equivalence relation (section 4). In both examples, without a mathematical bridge, linking the two mathematical representations of the case studies seemed impossible. 


\section{The Bridging Role of Mathematics at Work}

In this section, I provide an example of a bridging role of mathematics in biology. Biological phenomena such as the pattern formation of skin colors are often explained either functionally or mechanistically. Mechanistic explanations work by identifying the mechanisms responsible for the occurrence of the empirical phenomena (see Machamer et al. 2000). Biologists may also appeal to some functional features such as sexual selection or camouflage to explain the biological phenomena, but these functional explanations are beyond the scope of this paper. ${ }^{3}$

Mathematical biologists often explain the formation of the skin patterns of vertebrates by appealing to Turing equation models to capture reaction-diffusion (RD) mechanisms between biological cells. In his landmark paper, "The chemical basis of morphogenesis", Turing (1952) proposed a mechanistic explanation for the phenomenon of morphogenesis: the shapes in living organisms are generated through the RD system. ${ }^{4}$ An RD system uses a set of nonlinear ${ }^{3}$ I am not dismissing the extremely important functional explanations of evolutionary biology. Since in this paper I am interested in examining the roles of mathematics in explanations, I focus here on the mechanistic explanations as a grounding for the higher-level explanation of the phenomenon of interest: namely, why (from a mechanistic point of view) is there a particular pattern formation on the skin color?

${ }^{4}$ In his paper, Turing was chiefly motivated to discuss a mechanism by which the genes of a zygote may determine the anatomical structure of the resulting organism. His proposal was later developed and mathematically elaborated upon, to explain the mechanism of the formation of different skin patterns on animal skins by using the partial differential equations of the RD mechanisms. These became later known as Turing equations. For a philosophical discussion of this topic, see Kitcher (1999). 
continuous dynamical equations to represent the interactions between microscopic biological cells. Hence, Turing models play a representational role in explaining why does this particular skin color pattern occur? The models trace the activities and interactions between microscopic biological cells involved in formation of the patterns in any self-regulated system with an underpinning RD mechanism.

In the case of squamates (lizards and snakes), the interactions between different elements of chromatophore cells result in the dynamic formation of skin color patterns. ${ }^{5} \mathrm{RD}$ models then calculate the concentration of the pigmentary and structural elements at a given time, based on the substances' diffusion, feed rate, removal rate, and reactions between them. More details about the mathematics of the Turing patterns are provided in section 3.2.

In contrast to the Turing explanation, which appeals to the interactions among microscopic biological skin cells, Manukyan et al. (2017) study a case according to which the full explanation of the formation of the labyrinth color pattern on the skin of a species of lizard requires more than the proposed Turing mechanism. Their study is the first in biological research on the formation of animals' skin color patterns proposing a different mathematical model, that of a discrete cellular automaton, to that of Turing's equations. They show that for a species of lizard, known as the ocellated lizard, the macro-dynamics of the skin color pattern is represented by the dynamics between the mesoscopic skin scales, rather than microscopic biological cells. ${ }^{6}$ Mesoscopic skin scales are quasi-hexagons whose long diagonal is about 150-200 microns in a newborn individual, and about 1mm in an adult. Microscopic biological

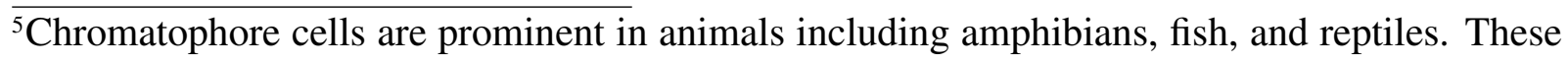
cells either contain pigments or reflect structures.

${ }^{6}$ Ocellated lizards (Timon lepidus) are primarily found in Southern Europe. The study is based on the analysis of time series of ocellated lizards over four years. 


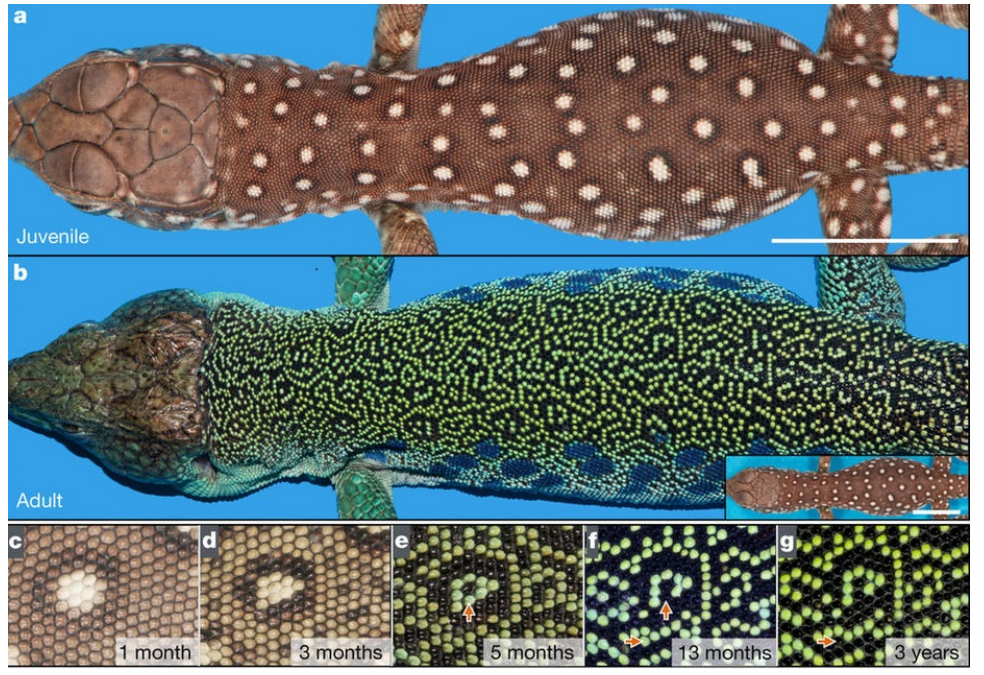

Figure 2: Change in the skin color patterns of ocellated lizards from Manukyan et al. (2017).

skin cells are typically 20 microns in size, and not visible to the naked eye. ${ }^{7}$ Figure 2 illustrates the changes in skin color patterns of the ocellated lizards at multiple time points over about three years, from juvenile (figure $2 a$ ) to adult (figure $2 b$ ).

Manukyan et al. (2017) claim that the units of the mesoscopic skin scale, rather than microscopic biological cells, establish the pattern formation of skin color in ocellated lizards. They show how the mesoscopic scale units can be modeled by a discrete cellular automaton that generates color patterns at the macroscopic scale of the skin of ocellated lizards. This seems to be fairly different to the Turing explanation according to which the microscopic skin scales, rather than mesoscopic biological cells, establish the color pattern. In this context, a natural puzzle for mathematical biologists arises: how can two distinct representations, the Turing model and the cellular automaton model, capture the dynamics of a single empirical phenomenon, the formation of color patterns on ocellated lizards? Take the explanandum as: ${ }^{7}$ I obtained the exact size of the different cell scales from Michel C. Milinkovitch, the leading author of the paper. 
there are two distinct descriptions for the formation of patterns on the skin of ocellated lizards. Before answering why this is the case, it is necessary to say a few words about the mathematics of cellular automata.

\subsection{Cellular Automaton Models as Higher-level Representations}

Cellular automaton models, originally developed by Von Neumann (1951), offer a mathematical characterization of the dynamics of various kinds of complex empirical and natural phenomena (see Wolfram 1984; Toffoli 1984; Langton 1986; Ermentrout and Edelstein-Keshet 1993 for an overview and examples). Roughly speaking, cellular automata are composed of a set of units (grids of elements) spanned over an ( $n$-dimensional) spatial structure. At time $t_{1}$, for a given cellular automaton, each unit is in a state $\sigma_{i}$ from a set $\Sigma$ of finitely many possible states. Each unit can only interact with units in its neighborhood, according to a set $R$, composed of deterministic or probabilistic rules. These rules specify how the state of a unit should change based on the structure of the states of its local neighbors. Time-steps in cellular automata are discretely incremented. At each incremental time-step $t_{2}, t_{3}, \cdots, t_{n}$, unit states are organized according to the instructions of $R$ relative to their local neighbors. The organization of the unit states continues by iterating on the set $R$. From these local interactions, a cellular automaton evolves into different kinds of macro-patterns over the whole spatial structure. The diachronic aggregation of the cellular automaton instructions for the state change of units of a grid gives rise to the emergence of various complex patterns at a macro-level.

Take the units of the cellular automaton to be the mesoscopic hexagon skin scales. The pattern formation of the skin color of ocellated lizards is generated by changes in influence 
dynamics of the quasi-hexagonal units of a probabilistic cellular automaton model. Consider the ocellated lizard skin as a spatially expanded grid of units, each element being a mesoscopic scale unit of skin. The set of states for the units of this cellular automaton are two colors, green and black. At birth, ocellated lizards have brown skin with white polka dots spread over it (as shown in figure 2). Within a few months, the skin pattern turns into arrays of black and green units; and the color pattern grows over their skin according to the dynamic computation of the color states of individual mesoscopic skin scales until the lizards reach the age of sexual maturity. During transition from juvenile to adult patterns, the skin color units flip between green and black according to some probabilistic rules over the quasi-hexagonal lattice of skin scales.

The color of the mesoscopic skin scales switches depending on the colors of the neighboring units. The general rules are as follows: with a very high probability, green units tend to exhibit four black and two adjacent green units; with a very high probability, black units tend to exhibit three green and three neighboring black units. ${ }^{8}$ Hence, formation of the skin color pattern on ocellated lizards invokes an appeal to cellular automaton models and mesoscopic skin units. This seems to be fairly different from the micro-level Turing explanation of the pattern formation in which microscopic biological cells, rather than the mesoscopic skin units, establish the pattern formation of skin color in ocellated lizards. The following question with respect to the formation of skin patterns arises: how does this cellular automaton pattern relate to the theoretical Turing explanation in mathematical biology? To answer, first let us briefly look at Turing models.

${ }^{8}$ The probabilistic distributions of the color transition rules for this cellular automaton model are derived from discrete RD numerical simulations. 


\subsection{Turing Models as Lower-level Representations}

In "The chemical basis of morphogenesis", Turing (1952) proposed a mechanistic explanation of morphogenesis in terms of RD systems. His main idea was that the formation of spatial patterns in living organisms can happen by interaction between two substances with different spreading rates. Turing showed that in certain systems, a homogeneous steady state is indeed unstable, and any small local deviation from this steady state (i.e., diffusion) is sufficient to trigger the beginning of pattern formation. Assume we only have two substances in a finite domain: activators, which produce more of themselves; and inhibitors, which slow down the production of activators. Diffusion as a stabilizing mechanism balances the amount of each.

The dynamic formation of skin color patterns in vertebrates such as zebrafish is known to be the result of microscopic non-linear interactions among pigment cells that obey the Turing equations. ${ }^{9}$ It is shown that a set of nonlinear partial differential equations gives a mechanistic explanation for the color pattern formation of zebrafish (Nakamasu et al. 2009). These equations reveal that only two types of choromatophore cells (melanophores and xanthophores) dominate the biological process of pattern formation. Manukyan et al. (2017) adapt this set of equations to formulate the color pattern formation on the skin of ocellated lizards. Consider the two variables $u$ and $v$ representing the densities of two kinds of pigment cells, melanophore and xanthophore, respectively; $w$ representing a long-range factor of diffusion; $F, G, H$ representing interactions among the chromatophore cells; $c_{u}, c_{v}$, and $c_{w}$ representing the coefficients for the decay processes; $D_{u} \nabla^{2} u, D_{v} \nabla^{2} v$, and $D_{w} \nabla^{2} w$ representing diffusion processes ( $D_{u}$ is the

${ }^{9}$ The micro-scale Turing explanation for such pattern formation is an approximation of the sustained micro-scale non-equilibrium dissipation, involving short- and long-range interactions among biological cells. 
diffusion coeffiecient and $\nabla^{2} u$ is the Laplacian). ${ }^{10}$ The following system of partial differential equations gives the two-dimensional representation of the skin color patterns of zebrafish: ${ }^{11}$

$$
\begin{aligned}
& \frac{\partial u}{\partial t}=F(u, v, w)-c_{u} u+D_{u} \nabla^{2} u, \frac{\partial v}{\partial t}=G(u, v, w)-c_{v} v+D_{v} \nabla^{2} v \\
& \frac{\partial w}{\partial t}=H(u, v, w)-c_{w} w+D_{w} \nabla^{2} w
\end{aligned}
$$

Call the micro-level description of the color pattern formation $L_{1}$ and these Turing models representing this descriptive level $M_{1} . M_{2}$ (as discussed in section 3.1.), is a discrete cellular automaton model representing the macroscopic pattern formation of the skin colors by referring to the mesoscopic skin scale units. Hence, there are two different kinds of models $M_{1}$ and $M_{2}$, at two different representational levels. But how can we get from the micro-level, the Turing model, to the macro-level, the cellular automaton representation? Why are there two very different representations for the same empirical phenomenon, the color pattern formation? How do these two representations relate?

To explain why the cellular automaton pattern is a plausible mathematical representation of the skin color pattern, we need to understand how the microscopic interactions among the biological cells translate into a cellular automaton pattern.

\subsection{From Turing Models to Cellular Automaton Patterns}

The case study presents the following explanatory gap: Given that pattern formation at the micro-level of biological cells can be represented by a set of differential equations, how can we ${ }^{10}$ The decay terms model cell behaviors such as division, differentiation, and death. The values of $c_{u}, c_{v}, c_{w}$ parameters are based on Nakamasu's model.

${ }^{11}$ The authors also consider boundary conditions on the functions $F, G, H$ to avoid any unrealistic production rate of the substances. 
explain the formation of cellular automaton patterns on the macro-level of the skin? Scientific intuition says there should be a way to fill this gap. To confirm this intuition in a stable and reliable way, Manukyan et al. (2017) appeal to a set of mathematical facts. To obtain the discrete $\mathrm{RD}$ models from the continuous ones requires considering the dual correspondence between Voronoi diagrams and Delaunay triangulation. ${ }^{12}$ Only after adding this duality fact to mathematical knowledge about continuous RD models, obtaining discrete RD models became possible. This duality is the bridge principle at work.

First, to obtain the discrete RD equations, Manukyan et al. (2017) approximate the continuous RD equations by discretization to edges of a square lattice (with edge length equal to $S$ and a sufficiently small edge width $\epsilon$ ). Discretization is such that the RD equations are essentially unchanged, with the same coefficients. The only difference with the continuous RD equations is the replacement of the Laplacian $\nabla^{2} u(x)$ by its discrete counterpart:

$\sum_{x^{\prime}}\left[u\left(x^{\prime}\right)-u(x)\right]$, where $x^{\prime}$ is the neighbor of $x$. The diffusion coefficient $D_{u}$ is replaced by a factor of $\epsilon^{-2} D_{u}$. Continuous RD equations on a Voronoi diagram approximate the lizard skin scales. Discrete RD equations on Delaunay triangulation are then obtained from the continuous $\mathrm{RD}$ equations. Consider $z$ denoting the center of a hexagon, and $z^{\prime}$ denoting the centers of the adjacent hexagons. The discrete Laplacian on the Delaunay triangulation becomes: $\nabla^{2} U(z)=$ $\sum_{z^{\prime}}\left[U\left(z^{\prime}\right)-U(z)\right] .{ }^{13}$ Then, they show that functions $U, V, W$ approximately satisfy the

${ }^{12}$ For a given set of discrete points $\mathrm{P}$ in a plane, a Delaunay triangulation is a triangulation such that no point of the given set is inside the circumcircle of any triangle obtained. A Voronoi diagram is a partitioning of a plane into regions based on distance to points $\mathrm{P}$ in a specific subset of the plane.

${ }^{13} U, V, W$ at the center $z$ of a hexagon are defined as the averages corresponding to $u, v, w$ on the vertices of the square lattice inside a hexagon. 
discrete RD equations on the Delaunay triangulation. The bridge principle, the mathematical fact concerning the transformation of the continuous RD equations on a Voronoi diagram to the discrete RD equations on the corresponding Delaunay triangulation, provides a sufficiency condition for obtaining the discrete RD equations from the continuous ones. Figure 3 illustrates this dual correspondence.

This example shows that the drawing of consequence step of the inferential framework is too simplistic to straightforwardly capture how some scientists such as Manukyan et al. (2017) use some pieces of mathematics, independent from any mathematical representations $M_{1}$ and $M_{2}$, to explain the link between the two different descriptions of an empirical phenomenon. Obtaining the discrete RD model from the continuous one is not just drawing consequences, in the sense of Bueno and Colyvan (2011, 353) and Bueno and French (2018, 52-53):

The second step consists in drawing consequences from the mathematical formalism, using the mathematical structure obtained in the immersion step. We call this step derivation. This is, of course, the key point of the application process, where consequences from the mathematical formalism are generated.

In the case study presented, it is epistemically impossible to obtain the discrete RD model without adding a new mathematical fact, the duality between Voronoi diagram and Delaunay triangulation, to the toolbox of scientists. The approximated RD models, therefore, are not simply the result of drawing mathematical consequences from the mathematical representation obtained in the immersion step (i.e., the continuous Turing equations). Here, an approximation procedure is at work. Why this approximation, rather than another? Because the scientists have the discrete cellular automaton model and want to link that discrete model to the continuous Turing equations in order to improve their understanding of the biological phenomena. 
One main reason is that manipulating formulas and directly drawing mathematical consequences does not always show what the scientists aim to explain. To connect these representations, something else, a previously unrelated piece of mathematics, is required. I call this piece a mathematical bridge. A mathematical bridge provides sufficient conditions that make obtaining a different mathematical representation of the same empirical phenomenon possible. ${ }^{14}$ The derivation step of Bueno and Colyvan (2011) and Bueno and French (2018) merely shows that a mathematical structure is a mathematical consequence of another. The case study illustrates that we cannot merely derive $M_{2}$ from $M_{1}$; rather, we need an additional fact, the mathematical bridge, that makes obtaining the $M_{2}$ possible. Therefore, the bridge is explanatory because it answers why and how the two descriptive levels of an empirical phenomenon connect.

In contrast to the drawing of consequences step of the inferential account, sometimes the scientific aim is not merely drawing mathematical consequences, and then to interpret these consequences back to a micro-level description of the phenomenon $L_{2}$. In some situations, we have two mathematical representations from two distinct kinds of scientific study, and then the main goal is to explain how one given mathematical representation links to another, and accordingly how the two descriptions of the empirical phenomenon under study relate. The second mathematical representation gives some hints as to what kinds of approximations we need in order to justify the link. These hints incorporate some pragmatic and occasionally messy and context-dependent considerations that motivate scientists' search for mathematical bridges.

${ }^{14}$ I do not claim that this mathematical piece is unique. In principle, there might be other ways to explain the link between the two descriptions of the empirical phenomenon. Nothing I say here rules out such alternatives. 


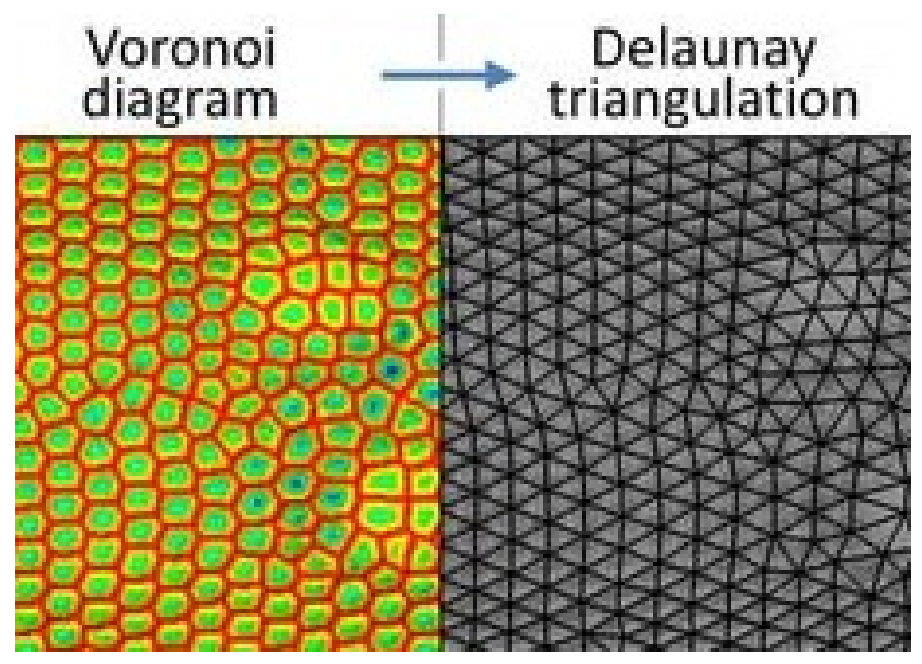

Figure 3: Voronoi tiling diagrams and Delaunay triangulation from Manukyan et al. (2017).

Obtaining the discrete RD model from the continuous one mathematically confirms biological intuitions of scientists about the presence of some new geometrical parameters at work responsible for the appearance of macroscopic cellular automaton patterns. Scientists then interpret the new parameters in the following way: the generation of the discrete RD mechanism is due to the dramatic difference of thickness between scale and inter-scale skin of the ocellated lizards.

Having the discrete RD models, Manukyan et al. (2017) then show that the cellular automaton behavior can be obtained when the diffusion coefficients in the system of discrete partial differential equations are reduced by a factor greater than $80 \%$ in the inter-scale regions. This approximation is validated with the help of computer simulations: that the discrete RD model and the cellular automaton have the same statistical properties. ${ }^{15}$ I have now enough ${ }^{15}$ Understanding how computer simulations factor into scientific explanations is beyond the scope of this paper. Interested readers are referred to Durán (2017) and Parker (2017) for some initial insights. The use of computer simulations in obtaining $M_{2}$ from $M_{1}$ additionally 
pieces at hand to revise the schema of the inferential account. This revised account is illustrated in figure 4.

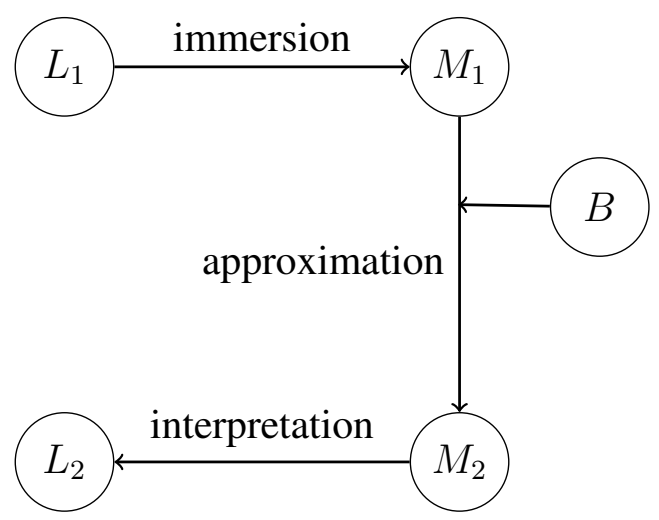

Figure 4: Bridging role of mathematics in inter-level explanation of color pattern formation.

In figure 4, the continuous RD model $M_{1}$ represents the mechanisms of the biological skin cells at the micro-level $L_{1}$. The macro-level skin pattern $L_{2}$ is represented by a cellular automaton model $M_{2}$. Scientists require some bridge principles to make sense of the relation between $M_{2}$ and $M_{1}$. In particular, $M_{2}$ cannot be derived from $M_{1}$ alone. To obtain $M_{2}$ requires a mathematical principle whose relation to $M_{1}$ and $M_{2}$ was previously unknown and which is not entailed by $M_{1}$. This bridging principle is the mathematical fact that Voronoi diagrams and Delaunay triangulations are dual $(B) . B$ is used in scientists' attempt to close the explanatory gap between the two descriptive levels of the empirical phenomenon of interest. $B$ provides mathematical possibility for obtaining $M_{2}$ from $M_{1}$. The crucial point to stress here is that what I am calling the bridge principle, is independent from either of the two models $M_{1}$ or $M_{2}$. Bueno and Colyvan (2011) and Bueno and French (2018), for instance, might want to say that $M_{2}$ is merely an extension of $M_{1}$ and is easily understood in terms of their partial models. challenges the claim that the derivation step (ii) of figure 1 is sufficient to explain the role of mathematics in the present case study. 
However, this is not the case because the bridge principle $B$ here, which is independent from either of the two models $M_{1}$ or $M_{2}$, is essential to relate them. Finding a relevant mathematical bridge can sometime be a significant achievement. Hence, the step (ii) of the inferential account, drawing of consequences, should be replaced by an approximation step which allows to choose a bridge principle and an approximation procedure.

The schema illustrated in figure 4 has the maximum amount of apparatus to capture the roles of mathematics in some explanations. Needless to say, not all steps illustrated here might manifest themselves in different instances of the applicability of mathematics to empirical phenomena. The revised schema captures the use of mathematical bridges in obtaining new mathematical representations. Moreover, it illustrates that in some cases, due to extreme levels of difficulty or the epistemic impossibility of drawing consequences from a mathematical formalism, approximation procedures substitute the strict mathematical derivation. In some simple cases, the approximation might be sharpened and become purely mathematical in terms of drawing of consequences; though it need not be the case. Hence, the schema presented in figure 4 is broader than the inferential account of Bueno and Colyvan (2011) and Bueno and French (2018).

Let me clarify a potential objection as to whether the revised schema in figure 4 is something that Bueno and Colyvan's (2011) and Bueno and French's (2018) framework cannot account for. Bueno and French (2018, Chapter 9) discuss how their account can accommodate highly idealized models such as renormalization group techniques that are claimed to play a genuine non-representational role in the explanation of phase transitions. Why do they play a non-representational role? In one of the most promising responses, Batterman (2009) claims that there is no correspondence between physical structures and divergent limits; hence, no structural similarity can relate the physical world to the mathematical model. To handle this 
non-representational role of mathematics, Bueno and French's (2018) solution is to keep the step (ii) drawing of consequences fixed. Instead, they extend the step (i) and (iii) of their account, the immersion and the interpretation steps, to iterated immersion and iterated interpretation steps (figure 5). This means that, as they claim, sometimes in order to make sense of the applicability of mathematics, first, there is a mapping between the physical structure and the mathematical structure; then, there is a second immersion step from the mathematical structure to another mathematical structure. In a similar vein, iterated interpretations happen in order to map the mathematical representation back to the empirical world. In this way, Bueno and French (2018) claim that their account can accommodate the non-representational role of infinite limits when they apply to the empirical phenomenon of phase transition. Could it be that this iterated inferential account also can capture the applicability of mathematics in the case of the skin color pattern example? Not so for two reasons.

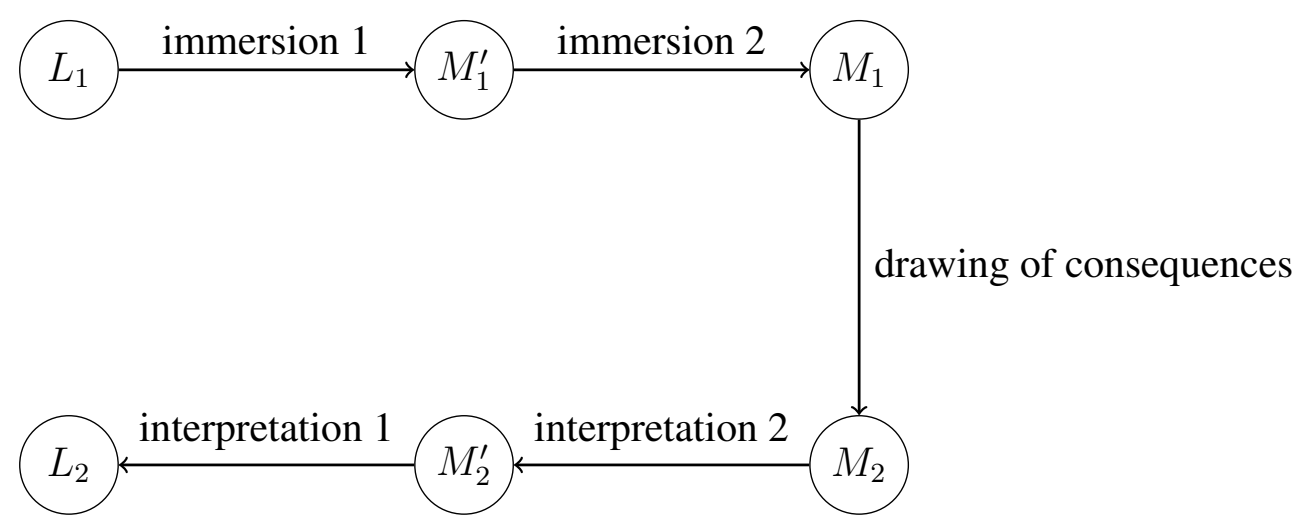

Figure 5: The iterated inferential account.

First, by their own definition, the immersion and the interpretation steps should capture the similarities between the physical and the mathematical phenomena. It is in virtue of this physical-mathematical similarity that the inferential conception of applied mathematics gets off the ground. However, establishing a relation between the two mathematical structures $M_{1}^{\prime}$ and 
$M_{1}$ is not really an immersion step, in the same way that the relation between $L_{1}$ and $M_{1}^{\prime}$ is. The structural similarity between $M_{1}^{\prime}$ and $M_{1}$ is purely mathematical, not physical-mathematical. As a result, the relation between $M_{1}^{\prime}$ and $M_{1}$ is not really immersion. In a similar vein, the relation between the two mathematical structures $M_{2}^{\prime}$ and $M_{2}$ is not really an interpretation step. The structural similarity between $M_{2}^{\prime}$ and $M_{2}$ is purely mathematical, not physical-mathematical. This is an objection as to whether the iterated inferential account really gets off the ground. Second, the iterated account does not open space for considerations of bringing new parts of mathematics to the mathematical toolbox of scientist for the explanations in question. In particular, it does not show the role of independent parts of mathematics when they make relating the two mathematical representations $M_{1}$ and $M_{2}$ possible.

The bridging role of mathematics as an explanatory role is compatible with a variety of ontological stances about mathematics. Here, I explore two major ontological views. Both views are committed to assigning a high status to the contribution of mathematics to scientific reasoning. First, at least partially, mathematics is embedded in and therefore constitutive of the empirical world (e.g., Bigelow 1988; Franklin 2014). If this is the case, scientific intuitions about the existence of a mathematical relation between the mechanistic explanations of the two levels is confirmed by mathematical bridges that are constitutive of the empirical world. Second, the mathematical bridges act as a piece of puzzle-filling in our incomplete schema of scientific reasoning. This view assigns a more instrumental, functional stance to mathematics. Relatedly, we might also expect that we will find a natural correspondence with the mathematical bridge in the future, as current scientific knowledge is evolving and by no means complete.

To summarize, first, in the study of the color pattern formation of vertebrates, mathematical biologists use continuous differential equations as a mathematical model for the representation 
of the interactions and activities among microscopic biological cells. In the formation of color patterns, a Turing model provides a mechanistic explanation for "why is there a specific kind of skin color pattern with reference to microscopic biological cells?" Second, a cellular automaton model is used to represent the formation of the color pattern at the macroscopic level, by making reference to the mesoscopic hexagonal cells. Third, the mathematical fact that Voronoi diagrams and Delaunay triangulations are dual acts as a bridge to obtain discrete RD models in explaining "why is there a cellular automaton model at the macro-level of the target phenomenon, given that the micro-dynamics between the biological skin cells correspond to a Turing model?" Without digging into some facts of mathematical geometry, the scientists could not unequivocally characterize the system, could not justify the presence of "an additional spatial parameter", and could not fully explain why we obtain the cellular automaton patterns from the continuous Turing models. Therefore, the bridging role of mathematics is an important role for mathematics in scientific explanations.

In the next section, I briefly discuss another interesting case from the history of science in which a mathematical bridge has made explaining the empirical adequacy of two mathematical representations possible. I will discuss the explanation of the empirically-significant, mathematical equivalence of matrix and wave mechanics as established by Von Neumann (1955). I have two reasons to discuss this case. First, Bueno and French (2018, Chapter 6) explore the exact same scientific case. Their discussion illustrates how mathematics unifies some apparently unrelated domains, such as quantum states, probability assignments, and logical inference. As I will argue, however, their discussion lacks sufficient resources to accommodate the essential role of the mathematical bridge, the Riesz-Fischer theorem in functional analysis, in establishing the empirically-significant, mathematical equivalence of matrix mechanics and wave mechanics. Second, this example will be known, at least in outline, 
to many readers. The details will nicely illustrate the mathematical bridge to relate the two mathematical models of quantum mechanics.

\section{Bridging Wave Mechanics and Matrix Mechanics}

Matrix mechanics is an algebraic approach, employing the techniques of matrix manipulation, for the representation of observable properties of quantum systems, such as position and momentum. Developed by Heisenberg (1925) and Born et al. (1926), matrix mechanics aims at providing a mathematical representation for quantum systems, that is as closely as possible to the mathematical formulations of classical mechanics; we must learn as much as possible about the behavior of quantum systems from the behavior of the Hamiltonian function. Matrix mechanics is articulated in a discrete space, and roughly assumes the following mathematical postulates for the representation of quantum phenomena. (1) The observable behavior of a quantum system, its position and its momentum, corresponds to time-dependent, Hermitian matrices Q and P, known as canonical matrices. (2) The canonical matrices satisfy the following quantum condition: $P Q-Q P=\frac{h}{2 \pi i} I$. (3) Equations of motions are $\dot{Q}=\frac{\partial H}{\partial P}$ and $\dot{P}=-\frac{\partial H}{\partial Q}$. (4) The Hamiltonian matrix $W=H\left(Q_{1}, \ldots, Q_{k}, P_{1}, \ldots, P_{k},\right)$ that represents energy is diagonal; otherwise, a canonical transformation matrix $S$ should be found such that $S^{-1} H S$ is diagonal. Finding solutions of quantum mechanical systems to the above representation has turned out to be complicated.

From an entirely different standing point, Schrödinger (1926a) used the mathematical machinery of differential equations, and developed wave mechanics to represent quantum systems. Wave mechanics has an underlying continuous space, and treats material particles as waves. A wave function $\psi(x)$ is associated with each particle, and describes the shape of the 
wave in three-dimensional Euclidean space. Wave mechanics, broadly, assumes the following mathematical postulates for the representation of quantum phenomena. (1) The position and momentum of a quantum phenomenon are represented by a wave operator, acting on the corresponding wave function. (2) Schrödinger's equation $\tilde{H} \psi=E \psi$ replaces the classical equation of motion. $\tilde{H}$ is obtained by substitution of $\mathrm{q}$ and $\mathrm{p}$ in the classical Hamiltonian by the following two operators: $\tilde{Q}=x$ and $\tilde{P}=-i \bar{h} \frac{\partial}{\partial x}$. The main wave-mechanical problem is then solving the partial differential equations.

As briefly shown above, these two representations of the quantum phenomena use very different mathematical apparatuses to illustrate quantum reality: matrix mechanics describes the quantum phenomena by discrete matrices and sums, whereas wave mechanics applies continuous functions and integration over those functions for this representation. Take the explanandum to be matrix and wave mechanics give empirically-significant, mathematically-equivalent representations of the quantum phenomena. The explanans is the mathematical proof that shows the empirically-significant, mathematical equivalence of these two representations. Schrödinger (1926b) aimed to show that the two mathematical representations of quantum phenomena, the wave and the matrix mechanics, were empirically equivalent. ${ }^{16}$ He wanted to show that the empirical equivalence can be explained in terms of a mathematical proof for the equivalence between the two mathematical representations. Schrödinger himself was not fully successful in achieving this goal, due to several conceptual and technical difficulties. ${ }^{17}$

\footnotetext{
${ }^{16}$ Around the same time, Eckart and Pauli also attempted to give similar equivalence proofs. I will not discuss this point in further details here, as Schrödinger's proof is the most elaborate one, with the highest historical influence.

${ }^{17}$ For a detailed characterization of this debate, see Muller (1997a) and Muller (1997b).
} 
On the other hand, using his Hilbert space formalism, Von Neumann (1955) characterized matrix mechanics with the totality of functions $F_{m}$, satisfying certain conditions. $F_{m}$ constructs the discrete space of matrix mechanics. In a similar vein, he identified the totality of functions $F_{w}$, satisfying certain conditions. $F_{w}$ constructs the continuous space of Schrödinger's wave mechanics. Then, he appealed to the Riesz-Fischer theorem in functional analysis to give the proof for the isometric isomorphism of $F_{m}$ and $F_{w} . F_{m}$ and $F_{w}$ are not arbitrary sets of functions. Indeed, Von Neumann (1955, pp. 30-31) emphasizes the empirical significance of $F_{m}$ and $F_{w}$ as follows: (i) these functions "... are the entities which enter most essentially into the problems of quantum mechanics", and (ii) they "... are the real analytical substrata of the matrix and wave theories." (i) and (ii) gives sufficient reasons to von Neumann to claim that "... this isomorphism must always yield the same numerical results." Therefore, the mathematical proof relates to making claims about the quantum phenomena. To put it differently, von Neumann gave a mathematical proof for the equivalence of wave and matrix mechanics which has empirical significance; that is, the mathematical equivalence of matrix and wave representations of quantum phenomena is understood in terms of the same numerical results that they provide. This empirical significance can be captured as follows: von Neumann's mathematical formulation of quantum mechanics describes the states of the physical system by Hilbert space vectors and the measurable quantities by Hermitian operators.

As Bueno and French (2018, Chapter 6) point out, von Neumann's mathematical proof of the theoretical equivalence of matrix and wave mechanics reveals how appropriate analogies and structural similarities between the two mathematical representations of quantum phenomena gave rise to the development of a more general framework, von Neumann's Hilbert space formalism. Bueno and French successfully show that their three-step representational framework captures the significance of structural similarities between the two mathematical 


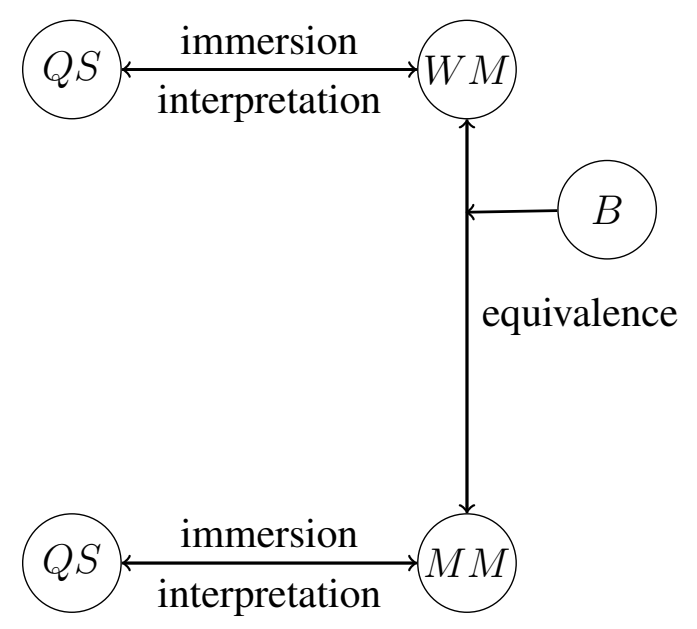

Figure 6: Bridging role of mathematics in explaining the equivalence of matrix and wave mechanics.

representations, and how such similarities motivated mathematicians to find a more general framework that unifies seemingly separate pieces of mathematics and logic. However, this framework does not have sufficient resources to reveal how a new piece of mathematics made the equivalence proof possible. Recall that their representational framework is composed of three steps: immersion, drawing of consequences, and interpretation. I maintain that the drawing of consequences cannot completely capture the role of mathematics in this quantum endeavour. Indeed, von Neumann did not claim that $F_{m}$ is obtained by a mathematical derivation from $F_{w}$, nor that $F_{w}$ is obtained by a consequence derivation from $F_{m}$. He used a new piece of mathematics from functional analysis, the Riesz-Fischer theorem, to give the proof of an empirically-significant, mathematical equivalence relation. I take the Riesz-Fischer theorem to be a mathematical bridge. The drawing of consequence step of Bueno and Colyvan's (2011) and Bueno and French's (2018) representational framework does not have sufficient resources to show how this mathematical bridge, independent from the two representations, makes the equivalence proof possible, since it requires bringing in outside 
considerations such as the Riesz-Fischer theorem from functional analysis. The importance of adding the bridge is that sometimes it changes the nature of the activity of drawing consequences. Figure 6 illustrates how the bridging role of mathematics (B) influences the explanation of the empirically-significant, mathematical equivalence of wave mechanics (WM) and matrix mechanics (MM) for a quantum system (QS). I want to stress once again a central point. The representational account does a fine job of modeling matrix mechanics and wave mechanics. But this account does not have sufficient resources to link the two, at least not directly. The mathematical bridge was provided by a further mathematical domain that had been perhaps known to some mathematicians but not to the physicists who eventually embraced it as a bridge. It might even have been a new mathematical approach whose development was in itself a mathematical achievement. In either case, it was not a mere corollary of the mathematical formalism used for the representation of the empirical phenomenon.

In summary, some mathematical bridges will be evident to the scientists working on the problem. Others might not be known to the scientists in question. They might have to consult their friendly neighborhood mathematician for suggestions. The mathematical contribution of Stanislav Smirnov, a Field medalist and a co-author of Manukyan et al. (2017), to the group of biologists is a clear example. He put insights about the dual relation between Voronoi diagrams and Delaunay triangulations on the table and so made possible the explanation of how and why the two representations (one in terms of differential equations and the other in terms of cellular automata) link. There is also the possibility that there is no bridge known to anyone. The bridge has yet to be discovered or invented. This was von Neumann's case. He had to come up with a new piece of mathematics to explain why and how two very different mathematical representations of quantum phenomena show empirically-significant, mathematical equivalence. 


\section{Conclusion}

In this paper, I have identified a distinct role for mathematics in scientific explanations, the bridging role, which has not been fully appreciated in the literature. This role illustrates how mathematics acts as a reliable connecting scheme in our explanatory reasoning about different representations of an empirical phenomenon. Different kinds of mathematical bridges are possible. A bridge might connect different levels of empirical phenomena (as in the biological case) or it might establish the equivalence of phenomena (as in the quantum mechanics case). Still others might be possible.

Moreover, I have discussed that this bridging role differs from both the genuinely constraining-explanatory role and the representational role. By providing two relevant case studies from mathematical biology and physics, I have argued how this role is not a trivial extension of Bueno and Colyvan's (2011) and Bueno and French's (2018) framework for the applicability of mathematics to empirical phenomena. I have shown that adding a bridge principle as an explanans provides sufficient conditions for making some approximations possible. Accordingly, I have proposed revised schema that captures some instances of scientific practice more accurately, and helps us to better understand the full spectrum of activities that constitute applied mathematics.

Once alerted to examples of mathematical bridges and to examples where they might fail, we will likely find lots more. For instance, the equivalence of Lagrangian and Hamiltonian mechanics comes to mind. Interesting questions will arise such as: How are they related? Are they really equivalent? If so, what kind of roles mathematics play in establishing this equivalence? If not, as North (2009) and Curiel (2013) argue, what weaker relation is at work between the two mathematical representations? Perhaps, equivalence is a strong kind of 
relation and other kinds of relations worth analyzing. And some of these questions might only be answered following considerable historical investigation. It could be that the idea of mathematical bridges will open up a large and important new field for philosophical investigation.

Acknowledgements. I am very grateful to James Robert Brown, Laura Franklin-Hall, Franz Huber, Nicolas Fillion, Neil Dewar, Mario Günther, and three anonymous reviewers for very helpful feedback on earlier drafts of this paper. I also thank audiences at Canadian Society for the History and Philosophy of Science in Vancouver and the British Society for the Philosophy of Science in Durham for valuable discussions and suggestions.

\section{References}

Batterman, R. W. (2009). "On the Explanatory Role of Mathematics in Empirical Science". The British Journal for the Philosophy of Science 61(1), 1-25.

Bigelow, J. (1988). The Reality of Numbers: A Physicalist's Philosophy of Mathematics. Oxford: Clarendon Press.

Born, M., W. Heisenberg, and P. Jordan (1926). "Zur Quantenmechanik II". Zeitschrift für Physik 35(8-9), 557-615.

Bueno, O. and M. Colyvan (2011). "An Inferential Conception of the Application of Mathematics". Noûs 45(2), 345-74. 
Bueno, O. and S. French (2018). Applying Mathematics: Immersion, Inference, Interpretation. Oxford: Oxford University Press.

Curiel, E. (2013). “Classical Mechanics Is Lagrangian; It Is Not Hamiltonian”. The British Journal for the Philosophy of Science 65(2), 269-321.

Durán, J. M. (2017). "Varying the Explanatory Span: Scientific Explanation for Computer Simulations". International Studies in the Philosophy of Science 31(1), 27-45.

Ermentrout, G. B. and Edelstein-Keshet (1993). "Cellular Automata Approaches to Biological Modeling”. Journal of Theoretical Biology 160(1), 97-133.

Franklin, J. (2014). An Aristotelian Realist Philosophy of Mathematics. London: Palgrave Macmillan.

Heisenberg, W. (1925). "Über Quantentheoretische Umdeutung Kinematischer und Mechanischer Beziehungen. Z. Physik 33, 879-93.

Kitcher, P. (1999). "The Hegemony of Molecular Biology". Biology and Philosophy 14(2), $195-210$.

Krantz, D., D. Luce, P. Suppes, and A. Tversky (1971). Foundations of Measurement, Vol. I: Additive and Polynomial Representations. San Diego: Academic Press.

Lange, M. (2009). Laws and Lawmakers: Science, Metaphysics, and the Laws of Nature. New York: Oxford University Press.

Lange, M. (2012). "What Makes a Scientific Explanation Distinctively Mathematical?". The British Journal for the Philosophy of Science 64(3), 485-511. 
Lange, M. (2017). Because Without Cause: Non-causal Explanations in Science and Mathematics. New York: Oxford University Press.

Langton, C. G. (1986). "Studying Artificial Life with Cellular Automata". Physica D: Nonlinear Phenomena 22(1-3), 120-49.

Machamer, P., L. Darden, and C. F. Craver (2000). “Thinking About Mechanisms”. Philosophy of science 67(1), 1-25.

Manukyan, L., S. A. Montandon, A. Fofonjka, S. Smirnov, and M. C. Milinkovitch (2017). “A Living Mesoscopic Cellular Automaton Made of Skin Scales”. Nature 544(7649), 173-79.

Muller, F. A. (1997a). “The Equivalence Myth of Quantum Mechanics_Part I”. Studies in History and Philosophy of Science Part B: Studies in History and Philosophy of Modern Physics 28(1), 35-61.

Muller, F. A. (1997b). “The Equivalence Myth of Quantum Mechanics-Part II”. Studies in History and Philosophy of Science Part B: Studies in History and Philosophy of Modern Physics 28(2), 219-247.

Nakamasu, A., G. Takahashi, A. Kanbe, and S. Kondo (2009). "Interactions Between Zebrafish Pigment Cells Responsible for the Generation of Turing Patterns”. Proceedings of the National Academy of Sciences 106(21), 8429-8434.

North, J. (2009). “The Structure of Physics: A Case Study”. The Journal of Philosophy 106(2), $57-88$.

Parker, W. S. (2017). “Computer Simulation, Measurement, and Data Assimilation”. The British Journal for the Philosophy of Science 68(1), 273-304. 
Pincock, C. (2004). “A Revealing Flaw in Colyvan's Indispensability Argument”. Philosophy of Science 71(1), 61-79.

Pincock, C. (2007). "A Role for Mathematics in the Physical Sciences”. Noûs 41(2), 253-275.

Schrödinger, E. (1926a). “Quantisierung als Eigenwertproblem. Annalen der physik 385(13), 437-90.

Schrödinger, E. (1926b). “Über das Verhältnis der Heisenberg-Born-Jordanschen Quantenmechanik zu der Meinem”. Annalen der Physik 384(8), 734-756.

Stewart, J. (2008). Calculus: Early Transcendentals (6 ed.). Belmont: Thomson Learning.

Toffoli, T. (1984). “Cellular Automata as an Alternative to (Rrather than an Approximation of) Differential Equations in Modeling Physics". Physica D: Nonlinear Phenomena 10(1-2), $117-27$.

Turing, A. M. (1952). “The Chemical Basis of Morphogenesis”. Bulletin of mathematical biology 52(1-2), 153-197.

Von Neumann, J. (1951). The General and Logical Theory of Automata. In Cerebral Mechanisms in Behavior: The Hixon Symposium, pp. 1-41. New York: John Wiley \& Sons.

Von Neumann, J. (1955). Mathematical Foundations of Quantum Mechanics: New Edition. Princeton, NJ: Princeton University Press.

Wolfram, S. (1984). “Cellular Automata as Models of Complexity”. Nature 311(5985), 419-424. 